\title{
Análise morfométrica em Thoracocharax stellatus (Kner, 1858) (Characiformes, Gasteropelecidae) proveniente de diferentes bacias hidrográficas Sul-americanas
}

\author{
Edson Lourenço da Silva ${ }^{1,3}$, Liano Centofante ${ }^{2}$ \& Carlos Suetoshi Miyazawa ${ }^{2}$ \\ ${ }^{1}$ Programa de Pós-Graduação em Ecologia e Conservação da Biodiversidade, \\ Instituto de Biociências, Universidade Federal de Mato Grosso - UFMT, \\ Av. Fernando Corrêa da Costa, s/n, CCBS-II, CEP 78060-900, Cuiabá, MT, Brasil \\ ${ }^{2}$ Laboratório de Citogenética Animal, Departamento de Biologia e Zoologia, Instituto de Biociências, \\ Universidade Federal de Mato Grosso - UFMT, \\ Av. Fernando Corrêa da Costa, s/n, CCBS-II, CEP 78060-900, Cuiabá, MT, Brasil \\ ${ }^{3}$ Autor para correspondência: Edson Lourenço da Silva, e-mail: ed.loren@uol.com.br
}

SILVA, E.L., CENTROFANTE, L. \& MIYAZAWA, C.S. Morphometrics analysis in Thoracocharax stellatus (Kner, 1858) (Characiformes, Gasteropelecidae) from different South American river basins. Biota Neotrop., 9(2): http://www.biotaneotropica.org.br/v9n2/en/abstract?article+bn01109022009.

\begin{abstract}
A free-size canonical variable analysis was made to investigate the morphological pattern of variation among different Thoracocharax stellatus populations in South American river basins: Araguaia-Tocantins and Paraguay Basins Rivers from Brazil, and Orinoco's basin in Venezuela. Distinction among the samples from Araguaia-Tocantins and Orinoco were observed, with overlap of these populations with the Paraguay's basin samples. The primarily characters responsible for this discrimination are head length and dorsal fin length. The mechanisms that can act in this geographic variation among T. stellatus populations are discussed.

Keywords: geographic variation, morphometry, canonical variables, silver hatchetfish
\end{abstract}

SILVA, E.L., CENTROFANTE, L. \& MIYAZAWA, C.S. Análise morfométrica em Thoracocharax stellatus (Kner, 1858) (Characiformes, Gasteropelecidae) proveniente de diferentes bacias hidrográficas Sul-americanas. Biota Neotrop., 9(2): http://www.biotaneotropica.org.br/v9n2/pt/abstract?article+bn01109022009.

Resumo: Foram utilizadas análises de variáveis canônicas livres de tamanho com o objetivo de investigar os padrões de variação morfológica entre populações de Thoracocharax stellatus em bacias hidrográficas Sul-Americanas: rios das bacias Araguaia-Tocantins e Paraguai do Brasil e Orinoco na Venezuela. Distinções entre as amostras do Araguaia-Tocantins e Orinoco foram observadas, com leve sobreposição dessas populações com as amostras da bacia do Paraguai. Os caracteres morfométricos que são os principais responsáveis por esta diversificação são comprimento da cabeça e comprimento da nadadeira dorsal. Os mecanismos que podem estar atuando nesta variação geográfica entre populações de $T$. stellatus são discutidos.

Palavras-chave: variação geográfica, morfometria, variáveis canônicas, papudinho. 


\section{Introdução}

Indivíduos de uma mesma espécie que habitam diferentes regiões geográficas ou são expostos a diferentes condições ambientais, freqüentemente exibem distintos fenótipos (Gould \& Johnston 1972, Brett 1979). Nestes casos, as barreiras geográficas e/ou ecológicas permitem o estabelecimento de traços nas populações que representam modelos apropriados para o estudo da plasticidade fenotípica das espécies (Molina et al. 2006).

A família Gasteropelecidae compreende um grupo de peixes neotropicais de pequeno porte, que inclui nove espécies distribuídas em três gêneros: Carnegiella Eigenmann 1909; Gasteropelecus Bloch, 1784 e Thoracocharax Fowler, 1906, ocorrendo no Panamá e em todos os países da América do Sul, exceto Chile (Weitzman \& Palmer 2003). Apesar do pequeno número de espécies, esta família apresenta muitos problemas taxonômicos relacionados à distinção das mesmas. Desta forma, considerando a sua ampla distribuição, análises mais acuradas de indivíduos de diferentes localidades, podem revelar a existência de espécies adicionais (Weitzman \& Weitzman 1982, Weitzman \& Palmer 1996, Weitzman \& Palmer 2003).

Um exemplo de estudo que demonstrou tais divergências foi o realizado por Géry (1977) com Carnegiella marthae Myers, 1927 que apresentou diferenças marcantes em alguns caracteres merísticos entre as populações isoladas provenientes dos rios Orinoco e Negro, bem como Amazônia Peruana e Rio Madeira. Segundo este autor, a forma típica dos rios Orinoco-Negro têm sido chamada de Carnegiella marthae marthae, e a da Amazônia Peruana é denominada Carnegiella marthae schereri.
Thoracocharax stellatus (Kner, 1858), objeto deste estudo, representa também um interessante modelo para avaliar possíveis diferenças entre populações isoladas uma vez que se trata da espécie mais amplamente distribuída dentre os gasteropelecídeos (Weitzman \& Palmer 1996, Netto-Ferreira et al. 2007), ocorrendo nas bacias hidrográficas dos rios Amazonas, Paraguai, Araguaia-Tocantins e Orinoco (Weitzman 1960). Desta forma, este trabalho compara amostras de populações isoladas de T. stellatus provenientes de rios que compõem os sistemas de bacias Araguaia-Tocantins e Paraguai, do Brasil, e Orinoco, da Venezuela, com o objetivo de verificar possíveis variações morfológicas entre estas, contribuindo para o entendimento da plasticidade fenotípica da espécie.

\section{Material e Métodos}

\section{Material examinado}

Os indivíduos de T. stellatus utilizados neste estudo são provenientes de cinco regiões distribuídas nas bacias hidrográficas dos rios Paraguai, Araguaia-Tocantins e Orinoco (Figura 1). Os exemplares originários da bacia do rio Paraguai provêm de três locais distintos: localidade de São Gonçalo (SG), município de Cuiabá, Mato Grosso (15 39' 9,96" S and 56 4' 8,62" O); Reserva Particular do Patrimônio Natural SESC Pantanal (SE), município de Barão do Melgaço, Mato Grosso (16 38' 55,0" S and 56 28'06,2” O); e município de Barra do Bugres (BB), Mato Grosso (15 4' 41,13"'S and 57 10' 55,64" O). Este material está depositado no Laboratório de Citogenética Animal, Universidade Federal do Mato Grosso, município de Cuiabá, Mato Grosso (LCA 018, LCA 023, LCA 028).
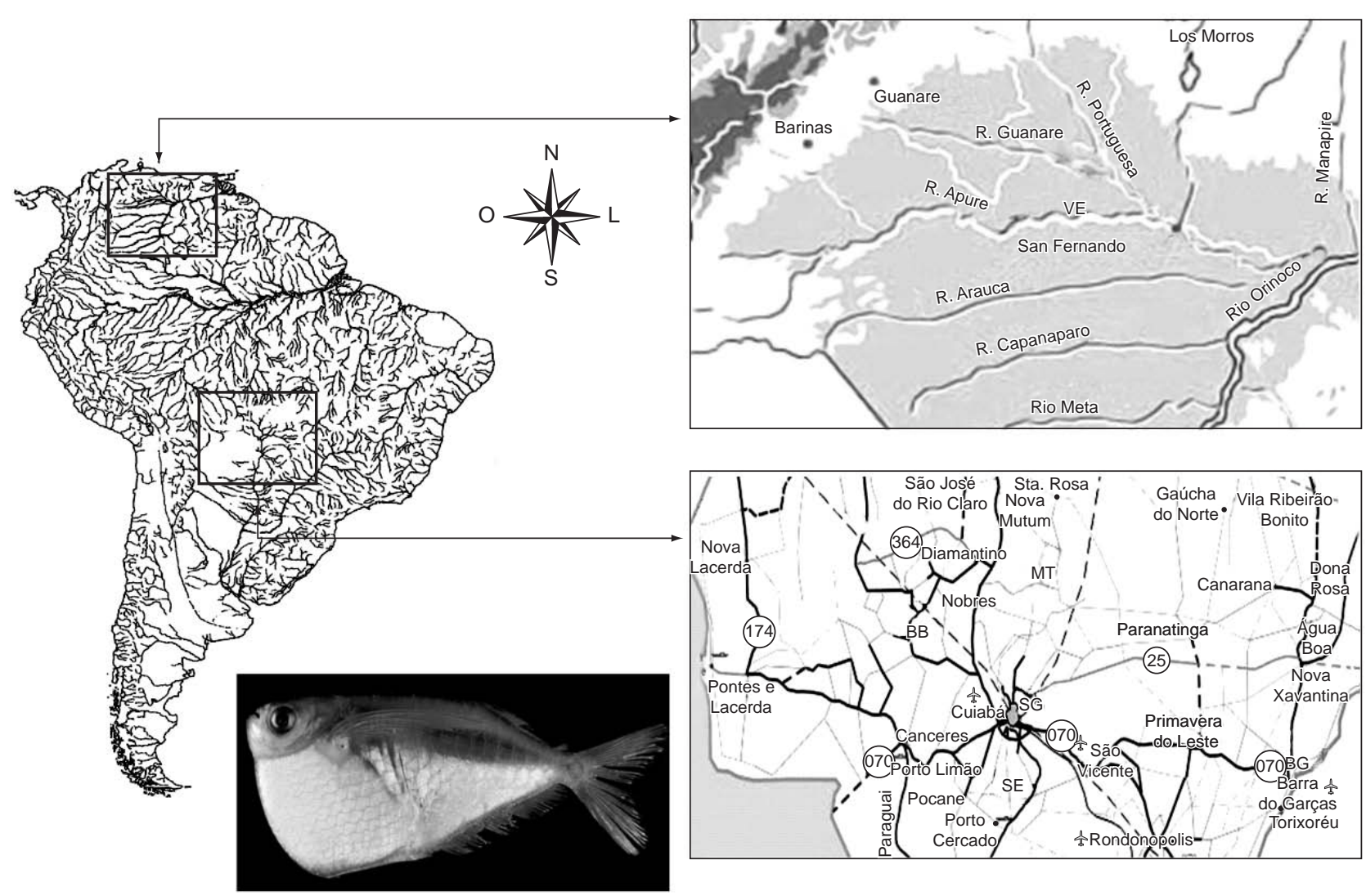

Figura 1. Mapa evidenciando a região de origem das cinco populações de Thoracocharax stellatus $(\mathrm{SG}=\mathrm{São}$ Gonçalo, $\mathrm{SE}=\mathrm{SESC}$ Pantanal, $\mathrm{BB}=\mathrm{Barra}$ do Bugres, $\mathrm{BG}=$ Barra do Garças e VE = Venezuela).

Figure 1. Map evidencing the region of origin of the five populations of Thoracocharax stellatus $(\mathrm{SG}=$ São Gonçalo, $\mathrm{SE}=\mathrm{SESC}$ Pantanal, $\mathrm{BB}=\mathrm{Barra}$ do Bugres, $\mathrm{BG}=$ Barra do Garças, and VE = Venezuela). 
Para o estudo morfométrico da população proveniente da bacia do Araguaia-Tocantins (BG) foram utilizados exemplares coletados no Rio Araguaia, município de Barra do Garças estado de Mato

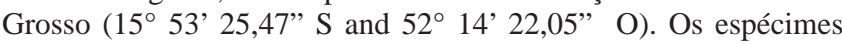
estão depositados no laboratório de Ictiologia do Departamento de Ciências Biológicas e da Saúde, Instituto Universitário do AraguaiaUniversidade Federal de Mato Grosso, Mato Grosso (ICLMA 015).

Os espécimes da bacia do Rio Orinoco são provenientes dos córregos Mamon e Maraca, município de Guanare, Estado Portuguesa - Venezuela (09 $04^{\circ} 18^{\prime \prime} \mathrm{N}$ and 69 30 ' $54^{\prime \prime}$ O; $09^{\circ} 04^{\prime} 18^{\prime \prime} \mathrm{N}$ and $69^{\circ} 30^{\prime} 4^{\prime \prime}$ O) e estão depositados no Alburn University Museum, Alburn Alabama - USA (AUM 22643, AUM 5299).

\section{Análises morfométricas}

Para análise morfométrica foram utilizados 73 indivíduos. Todas as medidas dos caracteres morfométricos foram obtidas com o auxílio de paquímetro de aço com precisão de $0,05 \mathrm{~mm}$. Medidas ponto a ponto foram realizadas para os seguintes caracteres: comprimento padrão $(\mathrm{CP})$, distância pré-dorsal (DPD), altura do corpo (AC), altura do pedúnculo caudal (APC), comprimento do pedúnculo caudal (CPC), comprimento da nadadeira dorsal (CND), comprimento da nadadeira peitoral (CNP), comprimento da cabeça (CC), comprimento do focinho $(\mathrm{CF})$, comprimento da maxila (CM), distância interorbital (DI) e diâmetro da órbita (DO), de acordo com Fink \& Weitzmann (1974) (Figura 2).

A forma do corpo foi estudada através da Análise de Variáveis Canônicas Livres de Tamanho (AVC) (Reis et al., 1990). Esta análise permite a visualização das similaridades morfológicas entre indivíduos pela projeção dos valores individuais para cada população sobre os eixos canônicos de um gráfico bidimensional, fornecendo valores de significância das diferenças nos valores médios dos caracteres morfométricos entre as populações de T. stellatus. As análises foram feitas utilizando-se o software PAST v. 1.3 (Hammer \& Harper 2004) e Systat v. 10 .

\section{Resultados}

As cinco populações de T. stellatus estudadas se sobrepõem consideravelmente em tamanho para todos os caracteres morfométricos (Tabela 1). Contudo, é possível verificar que as médias das populações do Araguaia-Tocantins e Paraguai são maiores que a população do

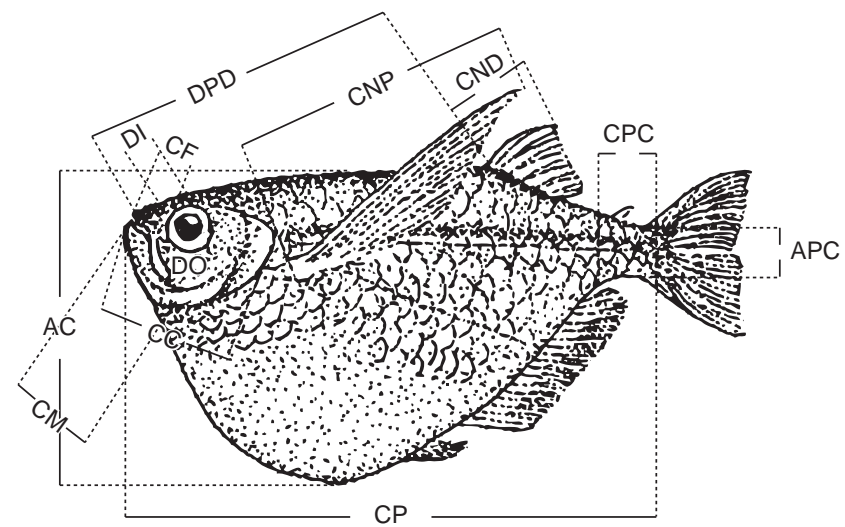

Figura 2. Caracteres morfométricos mensurados em Thoracocharax stellatus de cinco diferentes regiões. Comprimento padrão (CP), distância pré-dorsal (DPD), altura do corpo (AC), altura do pedúnculo caudal (APC), comprimento do pedúnculo caudal (CPC), comprimento da nadadeira dorsal (CND), comprimento da nadadeira peitoral (CNP), comprimento da cabeça (CC), comprimento do focinho (CF), comprimento da maxila (CM), distância interorbital (DI) e diâmetro da órbita (DO).

Figure 2. Morphometric characters measured in Thoracocharax stellatus of five different region: standard length (CP), pre-dorsal length (DPD), body height (AC), caudal peduncle height (APC), caudal peduncle length (CPC), dorsal fin length (CND), pectoral fin length (CNP), head length $(C C)$, snout length $(\mathrm{CF})$, upper jaw length (CM), interorbital distance (DI), and orbit diameter (DO).

Tabela 1. Média, desvio padrão (em parênteses) e amplitude de variação de 12 caracteres $(\mathrm{mm})$ para cinco populações de Thoracocharax stellatus. $(\mathrm{SG}=$ São Gonçalo, $\mathrm{SE}=$ SESC Pantanal, BB = Barra do Bugres, BG = Barra do Garças, VE = Venezuela $)$.

Table 1. Mean, standard deviation (in parentheses) and amplitude of variation of 12 characters $(\mathrm{mm})$ for five populations of Thoracocharax stellatus. (SG = São Gonçalo, SE = SESC Pantanal, BB = Barra do Bugres, BG = Barra do Garças, VE =Venezuela).

\begin{tabular}{|c|c|c|c|c|c|}
\hline \multirow[t]{2}{*}{ Caráter } & \multicolumn{3}{|c|}{ Paraguai } & \multirow{2}{*}{$\begin{array}{c}\text { Araguaia Tocantins } \\
\text { BG } \\
(\mathbf{N}=\mathbf{2 3}) \\
\end{array}$} & \multirow{2}{*}{$\begin{array}{c}\text { Orinoco } \\
\text { VE } \\
(\mathbf{N}=\mathbf{2 0})\end{array}$} \\
\hline & $\begin{array}{c}\text { SG } \\
(\mathrm{N}=\mathbf{2 6})\end{array}$ & $\begin{array}{c}\mathbf{S E} \\
(\mathbf{N}=27)\end{array}$ & $\begin{array}{c}\mathbf{B B} \\
(\mathbf{N}=\mathbf{2 0})\end{array}$ & & \\
\hline \multirow[t]{2}{*}{$\mathrm{CP}$} & $40,4(2,9)$ & $40,9(2,7)$ & $40,0(6,6)$ & $43,8(6,0)$ & $30,2(4,4)$ \\
\hline & $36,3-48,2$ & $36,1-47,5$ & $28,1-51,5$ & $27,8-55,0$ & $20,9-41,2$ \\
\hline \multirow[t]{2}{*}{ DPD } & $28,5(2,2)$ & $28,3(2,1)$ & $28,4(5,1)$ & $30,9(3,5)$ & $21,6(3,4)$ \\
\hline & $24,0-33,5$ & $24,9-32,6$ & $19,4-36,4$ & $25,9-37,7$ & $14,5-30,6$ \\
\hline \multirow[t]{2}{*}{$\mathrm{AC}$} & $24,1(1,8)$ & $24,3(1,4)$ & $23,6(4,4)$ & $25,5(2,8)$ & $17,1(2,7)$ \\
\hline & $21,1-28,6$ & $22,2-27,5$ & $16,9-30,5$ & $21,5-31,2$ & $10,9-23,9$ \\
\hline \multirow[t]{2}{*}{ APC } & $3,9(0,4)$ & $4,2(0,48)$ & $3,8(0,7)$ & $3,9(0,6)$ & $3,0(0,5)$ \\
\hline & $3,3-4,6$ & $3,3-5,2$ & $2,7-4,9$ & $2,8-5,0$ & $1,9-4,2$ \\
\hline \multirow[t]{2}{*}{ CPC } & $2,6(0,5)$ & $2,8(0,3)$ & $2,3(0,4)$ & $3,1(0,5)$ & $2,5(0,4)$ \\
\hline & $1,9-3,7$ & $2,2-3,5$ & $1,6-3,1$ & $2,4-4,3$ & $1,6-3,0$ \\
\hline \multirow[t]{2}{*}{ CPD } & $9,4(0,8)$ & $8,8(1,8)$ & $9,2(1,7)$ & $5,1(0,8)$ & $7,8(1,2)$ \\
\hline & $7,9-10,7$ & $5,0-13,3$ & $6,6-12,5$ & $4,0-7,0$ & $5,2-10,6$ \\
\hline \multirow[t]{2}{*}{$\mathrm{CNP}$} & $20,6(1,5)$ & $21,3(2,0)$ & $20,6(3,9)$ & $24,2(2,9)$ & $15,8(2,6)$ \\
\hline & $18-24,7$ & $18,2-25,7$ & $14,0-28,0$ & $19,1-29,6$ & $10,3-22,1$ \\
\hline \multirow[t]{2}{*}{$\mathrm{CC}$} & $9,7(0,7)$ & $9,7(0,8)$ & $9,8(1,7)$ & $9,3(1,3)$ & $8,9(1,5)$ \\
\hline & $8,4-11,2$ & $8,6-11,2$ & $7,3-13,3$ & $7,3-12,2$ & $5,7-12,6$ \\
\hline \multirow[t]{2}{*}{$\mathrm{CF}$} & $2,6(0,3)$ & $2,5(0,3)$ & $2,5(0,5)$ & $3,1(0,4)$ & $2,1(0,4)$ \\
\hline & $1,9-2,9$ & $1,9-3,3$ & $1,9-3,7$ & $2,4-3,9$ & $1,6-3,1$ \\
\hline \multirow[t]{2}{*}{$\mathrm{CM}$} & $7,3(0,9)$ & $6,6(1,1)$ & $8,1(1,3)$ & $7,1(0,9)$ & $4,2(0,8)$ \\
\hline & $5,6-8,6$ & $4,0-7,9$ & $5,3-10,3$ & $5,2-8,6$ & $3,0-7,1$ \\
\hline \multirow[t]{2}{*}{ DI } & $4,7(0,3)$ & $4,6(0,3)$ & $4,5(0,7)$ & $5,1(0,6)$ & $3,5(0,5)$ \\
\hline & $4,2-5,5$ & $4,2-5,3$ & $3,6-6,1$ & $4,0-6,3$ & $2,5-4,7$ \\
\hline \multirow[t]{2}{*}{ DO } & $3,6(0,2)$ & $3,5(0,3)$ & $3,4(0,5)$ & $3,8(0,4)$ & $2,9(0,4)$ \\
\hline & $3,2-4,0$ & $3,1-4,1$ & $2,4-4,5$ & $3,1-4,6$ & $2,2-3,8$ \\
\hline
\end{tabular}


Orinoco. As três sub-populações que compõem o grupo da bacia do Paraguai (SG, SE, e BB) apresentam um conjunto de caracteres com valores intermediários em comum. A amplitude de variação dos caracteres morfométricos de T. stellatus de todas as populações analisadas indicam que a variação intrapopulacional é bastante elevada.

Com a análise discriminante multivariada pôde-se constatar que há uma diferenciação significativa entre as populações com relação às médias dos caracteres morfométricos (Wilk's $\Lambda=0,0068 ; \mathrm{F}=21,34$; $\mathrm{p}<0,0001)$. A primeira variável canônica livre de tamanho (VC1) acumula $66,32 \%$ da variação observada entre os grupos, enquanto que a segunda $28,35 \%$. Tanto $\mathrm{VC} 1$ quanto $\mathrm{VC} 2$ possui valores negativos e positivos, demonstrando a interação entre forma e tamanho dos espécimes (Tabela 2). A ordenação das cinco populações sobre o eixo da VC1 mostra que há completa discriminação entre as populações do Araguaia-Tocantins e Orinoco (BG e VE respectivamente) (Figura 3b).

Contudo, as populações Araguaia-Tocantins em um extremo do espaço canônico e Orinoco em outro apontam discreta sobreposição com a população SE proveniente da bacia do Paraguai. No espaço produzido pela VC2 é possível verificar que a população do Orinoco (VE) se distingue claramente do conjunto formado pela população SG e BB (Paraguai) e que a população Araguaia-Tocantins se distingue somente da população BB (Paraguai) (Figura 3b).

A correlação dos caracteres originais com a VC1 mostra que o comprimento da nadadeira dorsal da população Araguaia-Tocantins e o comprimento da cabeça da população Orinoco contribuíram para a discriminação entre as populações (Figura 3a), sugerindo uma variação adaptativa local. No eixo da VC2, o comprimento do maxilar e a altura do corpo para a população Orinoco foram os caracteres mais importantes para discriminação das populações no espaço projetado pelas variáveis canônicas independentes do tamanho (Figura 3a).

\section{Discussão}

As análises morfométricas permitiram agrupar as diferentes populações de T. stellatus em pelo menos três conjuntos baseados em diferenças significativas tanto no tamanho quanto na forma dos indivíduos. Os grupos formados compreendem respectivamente as populações das bacias dos rios Araguaia-Tocantins, Paraguai e Orinoco.

Tabela 2. Coeficientes das variáveis canônicas discriminantes livres de tamanho (VC1 e VC2) de 12 caracteres morfométricos das populações de Thoracocharax stellatus. Os valores estão expressos como vetores de correlação entre os coeficientes e os caracteres originais.

Table 2. Coefficients of free-size canonical variable (VC1 and VC2) of 12 morphometrics characters of the populations of Thoracocharax stellatus. The values are expressed as correlation's vectors between the coefficients and original characters.

\begin{tabular}{crr}
\hline Caráter & VC1 & VC2 \\
\hline CP & 0,207 & $-0,022$ \\
DPD & 0,271 & 0,153 \\
AC & $-0,014$ & $-0,418$ \\
APC & 0,143 & $-0,125$ \\
CPC & $-0,039$ & 0,374 \\
CND & 0,761 & $-0,150$ \\
CNP & $-0,251$ & 0,242 \\
CC & 0,419 & 0,359 \\
CF & $-0,136$ & 0,259 \\
CM & $-0,102$ & $-0,513$ \\
DI & $-0,121$ & 0,022 \\
DO & $-0,001$ & 0,324 \\
\% variação & $66,32 \%$ & $28,35 \%$ \\
\hline
\end{tabular}

As variáveis que determinaram o tamanho e a forma dos indivíduos da população do Orinoco foram responsáveis pela distinção deste grupo dos demais. De forma geral, nesta população, caracteres como comprimento pré-dorsal, comprimento padrão, comprimento da cabeça e comprimento do maxilar foram os que apresentaram os menores valores médios. Na população do Araguaia-Tocantins somente o comprimento médio da nadadeira dorsal é menor que nas demais populações.

O exame da contribuição de cada caracter morfométrico isolado não implicou na diferenciação das populações de T. stellatus. Pelo contrário, caracteres como comprimento da cabeça e comprimento da
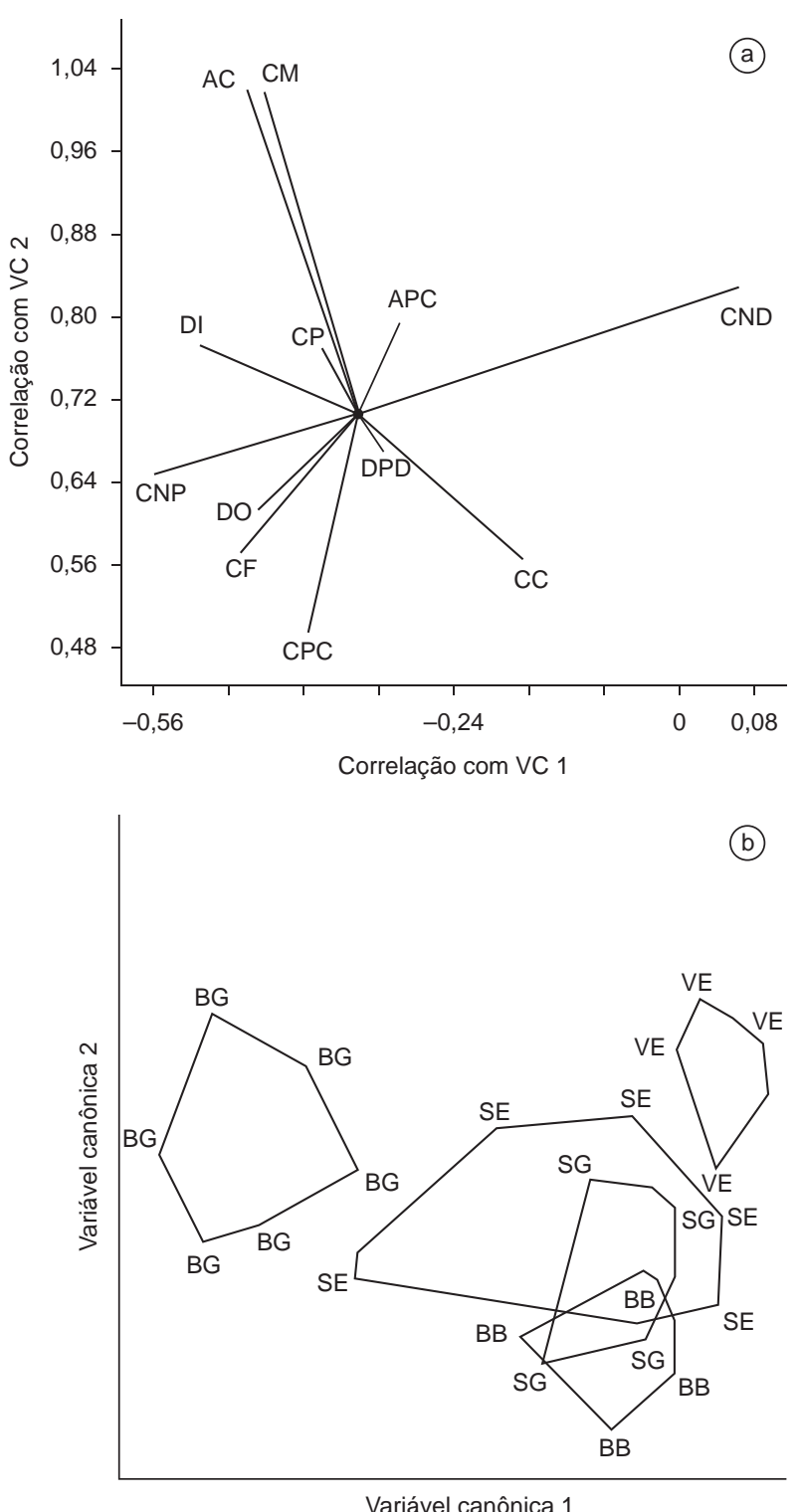

Figure 3. a) Vetores mostrando a direção da variação dos caracteres no plano da variável canônica 1 e 2; b) Diagrama de dispersão dos escores individuais das cinco populações de Thoracocharax stellatus no espaço definido pelas variáveis canônicas 1 e $2(\mathrm{SG}=$ São Gonçalo, $\mathrm{SE}=\mathrm{SESC}$ Pantanal, $\mathrm{BB}=$ Barra do Bugres, $\mathrm{BG}=$ Barra do Garças e VE =Venezuela).

Figura 3. a) Vectors showing the directions of characters on canonical variable 1 and 2; b) Diagram of dispersion of individual scores of the five Thoracocharax stellatus populations in the space defined for canonical variables 1 and 2 ( $\mathrm{SG}=$ São Gonçalo, $\mathrm{SE}=$ SESC Pantanal, $\mathrm{BB}=$ Barra do Bugres, $\mathrm{BG}=$ Barra do Garças, and VE = Venezuela). 
nadadeira dorsal juntos, foram responsáveis pela discriminação das populações do Araguaia-Tocantins e Orinoco no espaço da variável canônica 1. Já a altura do corpo e comprimento do maxilar foram os mais importantes na discriminação da população do Orinoco na variável canônica 2.

Embora a análise de caracteres morfométricos seja útil na identificação de amostras de diferentes rios, mesmo que estes estejam geograficamente próximos (Hermida et al. 2005), a considerável sobreposição dos valores dos caracteres individuais das populações que compõem a Bacia do rio Paraguai (SE, SG e BB), não permitiu a distinção destas sub-populações. Este fato permite sugerir que, até o momento, a distância entre as sub-populações desta bacia, não se configura como um mecanismo de isolamento a ponto de distinguir morfologicamente as populações por meio desta metodologia.

Em comparação com outros vertebrados, os peixes demonstram maior variação nos caracteres morfológicos tanto dentro quanto entre populações (Dunham et al. 1979, Allendorf 1988, Thompson 1991, Wimberger 1992). Nestes casos, a utilização da análise de variáveis canônicas livres de tamanho é interessante porque a elevada amplitude de variação dos caracteres intrapopulacionais em função, por exemplo, de dimorfismo sexual em tamanho ou estágios de desenvolvimento diferenciados, poderia mascarar as diferenças reais entre as populações (Reis et al. 1990).

Diferenças morfológicas entre populações geograficamente isoladas como as verificadas para $T$. stellatus têm sido freqüentemente relatadas na diferenciação de outros grupos de peixes neotropicais (Bemvenuti 2000, Shibatta \& Hoffmann 2005, Shibatta \& Artoni 2005). Nesta classe, a forma do corpo exerce in uência sobre a eficiência de locomoção em diferentes ambientes, particularmente no forrageamento e fuga de predadores (Neves \& Monteiro 2003). Assim, o estabelecimento de um caracter adaptativo que aperfeiçoe tais comportamentos pode ser decisivo para a persistência da população no local.

Os resultados encontrados foram baseados em séries comparativas de indivíduos de diversos tamanhos que possivelmente incluiu indivíduos de diferentes idades. Entretanto, essa grande amplitude de variação foi perfeitamente superada pela metodologia de análise utilizada. Mesmo assim, devido ao tamanho reduzido e diferenciado das amostras e a ausência de dados sobre populações intermediárias entre os pontos amostrados, é possível que as diferenças fenotípicas observadas entre os espécimes de T. stellatus não re itam a estrutura fenotípica da população natural. Dessa forma, demonstrações conclusivas de diferenças fenotípicas entre populações desta espécie requerem a adição de amostras de localidades intermediárias.

Assumindo que a notável diferenciação fenotípica entre populações de T. stellatus não resulta de amostragens insuficientes, existem pelo menos duas hipóteses para explicar os resultados encontrados: a) as condições ambientais diversas dirigem a diferenciação fenotípica; b) o isolamento das populações favorece alterações genéticas que implicam em mudanças fenotípicas.

Dentre os fatores ambientais diretamente relacionados às alterações fenotípicas destacam-se os efeitos da velocidade da água (Claytor et al. 1991, McLaughlin \& Grant 1994, Imre et al. 2002), da formação de microhabitats (Lundberg \& Stager 1985, Layzer \& Clady 1987, O’Reilly \& Horn 2004) e dos gradientes de temperatura (Hubbs 1922, Barlow 1961, Beacham 1990). Já com relação ao isolamento populacional, as alterações genéticas podem ocorrer principalmente por meio de mecanismos de isolamento por distância, isolamento geográfico, bem como eventos históricos (Prioli et al. 2002, Venere et al. 2007).

Os resultados deste trabalho mostram que os caracteres associados com a alimentação (comprimento da cabeça e comprimento do maxilar) e nado (altura do corpo e comprimento da nadadeira dorsal) foram os mais variáveis e capazes de distinguir as populações, sugerindo ação de diferentes regimes seletivos sobre estas.

Neves \& Monteiro (2003) observaram que a divergência morfológica intra-específica em Poecilia vivipara Bloch \& Schneider, 1801 está fortemente associada ao habitat. Nestas populações o corpo é mais alto em indivíduos que habitam regiões cobertas por macrófitas e mais fusiforme em indivíduos que habitam regiões abertas. O mesmo padrão pode ser observado para Bryconops caudomaculatus (Günther 1864) e Biotodoma wawrini (Gosse 1963), que apresentam corpo mais fusiforme quando encontrados em canais do que em lagoas da planície de inundação venezuelana (Langerhans et al. 2003).

No que diz respeito à morfologia de caracteres relacionados ao hábito alimentar, muitos autores afirmam que estas variações podem ser observadas mesmo entre peixes de uma mesma espécie, que ocupam diferentes ambientes, sendo uma resposta adaptativa ao tipo de alimento explorado (Lindsey 1981, Meyer 1990, Turigan et al. 1995).

Estudando o hábito alimentar de T. stellatus em um trecho lêntico da bacia do alto Rio Tocantins, Netto-Ferreira et al. (2007) observaram a estreita relação da espécie com a área próxima às margens, onde presas, na sua maioria invertebrados terrestres, são fornecidas pela vegetação ripária associada. A manutenção da relação terra-água no caso de um ambiente lótico, exigiria adaptações que maximizassem a captura dos alimentos levando, além de alterações comportamentais, à mudanças em aspectos morfológicos.

As divergências morfológicas observadas neste estudo entre as populações de T. stellatus provenientes das bacias dos Rios Orinoco, Araguaia-Tocantins e Paraguai são condizentes com a distância entre cada uma delas, uma vez que as amostragens foram feitas entre extremos de distribuição.

Com isso, estes resultados permitem sugerir a existência de considerável plasticidade adaptativa neste grupo que pode ocorrer, por exemplo, em função dos diferentes ambientes explorados e ausência de uxo gênico entre estes. Entretanto, a ausência de informações sobre populações localizadas em pontos intermediários aos estudados não permite inferir sobre a existência de variação clinal nos caracteres morfométricos que podem ser in uenciados por algum fator ambiental. Análises genéticas com marcadores moleculares em indivíduos destas localidades podem enriquecer as discussões sobre as observações deste trabalho ampliando assim, o conhecimento sobre a plasticidade T. stellatus.

\section{Agradecimentos}

Os autores são gratos a CAPES, CNPq, FAPEMAT e UFMT pelo suporte financeiro, ao Professor Donald Taphorn pelo auxílio com os dados da bacia do Orinoco, ao Professor Paulo Venere com os dados da bacia do Araguaia-Tocantins.

\section{Referências Bibliográficas}

ALLENDORF, F.W. 1988. Conservation biology of fishes. Conserv. Biol. 2:145-148.

BARLOW, G.W. 1961. Causes and significance of morphological variation in fishes. Syst. Zool. 10:105-117.

BEACHAM, T.D. 1990. A genetic analysis of meristic and morphometrics variation in chum salmon (Onchorhynchus keta) at three different temperatures. Can. J. Zool. 68(2):225-229.

BEMVENUTI, M.A. 2000. Diferenciação geográfica do peixe-rei Odontesthes argentinensis (Atherinopsidae) no extremo sul do Brasil, através da morfometria multivariada. Rev. Atlântica. 22:71-79.

BRETT, J.R. 1979. Environmental factors and growth. In Fish physiology (W.S. Hoar, D.J. Randall \& J.R. Brett, eds.). Academic Press, New York, v. 8 , p. $599-675$. 
CLAYTOR, R.R., MAc-GRIMMON, H.R. \& GOTS, B.L. 1991. Continental and ecological variance components of European and North American Atlantic salmon (Salmo salar) phenotypes. Biol. J. Linn. Soc. 44(3):203-229.

DUNHAM, A.E., SMITH, G.R. \& TAYLOR, J.N. 1979. Evidence for ecological character displacement in western American catostomid fishes. Evolution. 33(3):877-896.

FINK, W.L. \& WEITZMANN, S.H. 1974. The so-called cheirodontin fishes of Central America with description of two new species (Pisces, Characidae). Smithson. contrib. zool. 172:1-46.

GÉRY, J. 1977. Characoids of the world. T.F.H. Publications Inc., Neptune City.

GOULD, S.J. \& JOHNSTON, R.F. 1972. Geographic variation. Annu. Rev. Ecol. Syst. 3:457-498.

HAMMER, O. \& HARPER, D.A.T. 2004. Past version 1.3. Disponível em: 〈http://folk.uio.no/ohammer/past>. (último acesso em 18/09/2007).

HERMIDA, M., FERNANDEZ, J.C., AMARO, R. \& MIGUEL, S.E. 2005. Morphometric and meristic variation in Galician threespine stickleback populations, northwest Spain. Environ. Biol. Fish. 73(2):189-200.

HUBBS, C.L. 1922. Variations in the number of vertebrae and other meristic characters of fishes correlated with the temperature of water during development. Am. Nat. 56:360-372.

IMRE, I., McLAUGHLIN, R.L. \& NOAKES, D.L.G. 2002. Phenotypic plasticity in brook charr: changes in caudal fin induced by water ow. J. Fish Biol. 61(5):1171-1181.

LANGERHANS, R.B., LAYMAN, G.A., LANGERHANS, A.K. \& EEWITT, T.J. 2003. Habitat-associated morphological divergence in two Neotropical fish species. Biol. J. Linn. Soc. 80(4):689-698.

LAYZER, J.B. \& CLADY, M.D. 1987. Phenotypic variation of young-ofyear bluegills (Lepomis macrochirus) among microhabitats. Copeia. 3:702-707.

LINDSEY, C.C. 1981. Stocks are chameleons: plasticity of gill rakers of coregonid fishes. Can. J. Fish. Aquat. Sci. 38(1):1497-1506.

LUNDBERG, J.G. \& STAGER, J.C. 1985. Microgeographic diversity in the neotropical knife-fish Eigenmannia macrops (Gymnotiformes, Sternopygidae). Environ. Biol. Fish. 13(3):173-181.

McLAUGHLIN, R.L. \& GRANT, J.W.A. 1994. Morphological and behavioral differences among recently-emerged brook char, Salvelinus fontinalis, foranging in slow-vs. fast-running water. Environ. Biol. Fish. 39(3):289-300.

MEYER, A. 1990. Ecological and evolutionary aspects of the trophic polymorphism in Cichlasoma citrinellum (Pisces, Cichlidae). Biol. J. Limn. Soc. 39(3):279-299.

MOLINA, W.F., SHIBATTA, O.A. \& GALETTI Jr., P.M. 2006. Multivariate morphological analyses in continental and island populations of Abudefduf saxatilis (Linnaeus) (Pomacentridae, Perciformes) of Western Atlantic. PANAMJAS. 1(2):49-56.

NETTO-FERREIRA, A.L., ALBRECHT, M., NESSIMIAN, J.L. \& CARAMASCHI, E.P. 2007. Feeding habits of Thoracocharax stellatus (Characiformes: Gasteropelecidae) in the upper rio Tocantins, Brazil. Neotrop. Ichthyol. 5(1):69-74.
NEVES, F.M. \& MONTEIRO, L.R. 2003. Body shape and size divergence among populations of Poecilia vivipara in coastal lagoons of south-easten Brazil. J. Fish. Biol. 63(4):928-941.

O'REILLY, K.M. \& HORN, M.H. 2004. Phenotypic variation among populations of Atherinops affinis (Atherinopsidae) with insights from a geometric morphometric analysis. J. Fish. Biol. 64(4):1117-1135.

PRIOLI, S.M.A.P., PRIOLI, A.J., JÚLIO Jr., H.F., PAVANELLI, C.S., OLIVEIRA, A.V., CARRER, H., CARRARO, D.M. \& PRIOLI, L.M. 2002. Identification of Astyanax altiparanae (Teleostei, Characidae) in the Iguaçu river, Brazil, based on mitochondrial DNA and RAPD markers. Gen. Mol. Biol. 25(4):421-430.

REIS, S.F., PESSÔA, L.M. \& STRAUSS, R.E. 1990. Application of size-free canonical discriminant analysis to studies of geographic differentiation. Braz. J. Gen. 13(3):509-520.

SHIBATTA, O.A. \& ARTONI, R.F. 2005. Sobre a identidade das populações alopátricas de Astyanax (Characiformes, Characidae) das formações Furna 1 e Furna 2 do Parque Estadual de Vila Velha, Ponta Grossa, Paraná, Brasil. Publication UEPG: Biological and Health Science. 11(2):7-12.

SHIBATTA, O.A. \& HOFFMANN, A.C. 2005. Variação geográfica em Corydoras paleatus (Jenyns) (Siluriformes, Callichthyidae) do sul do Brasil. Rev. Bras. de Zool. 22(2):366-371.

THOMPSON, J.D. 1991. Phenotypic plasticity as a component of evolutionary change. Trends Ecol. Evol. 6(8):246-249.

TURIGAN, R.G., WAINWRIGHT, P.C. \& HENSLEY, D.A. 1995. Interpopulation variation in prey use feeding biomechanics in Caribbean triggerfishes. Oecologia. 102(3):296-304.

VENERE, P.C., SOUZA, I.L., MARTINS, C. \& OLIVEIRA, C. 2007. Occurrence of ZZ/ZW sex chromosomes in Thoracocharax stellatus fish (Characiformes, Gasteropelecidae) from the Araguaia River, South America. Genetica. 133(2):109-112.

WEITZMAN, S.H. 1960. Further notes on the relationships and classification of the South American characoid fishes of the subfamily Gasteropelecinae. Stanford Ichthyol. Bull. 7(4):217-239.

WEITZMAN, S.H. \& WEITZMAN, M. 1982. Biogeography and evolutionary diversification. In Neotropical freshwater fishes, with comments on the refuge theory (G.T. Prance, ed.). Columbia University Press, New York, p. 403-422.

WEITZMAN, S.H. \& PALMER, L. 1996. Do freshwater hatchetfishes really y? Trop. F. Hob. 45:195-206.

WEITZMAN, S.H. \& PALMER, L. 2003. Family Gasteropelecidae. In Checklist of the freshwater fishes of South and Central America (R.E. Reis, S.O. Kullander \& C. Ferraris Jr., eds.). Edipucrs, Porto Alegre, p. 101-103.

WIMBERGER, P.H. 1992. Plasticity of fish body shape, the effects of diet, development, family and age in two species of Geophagus (Pisces: Cichlidae). Biol. J. Linn. Soc. 45(3):197-218.

Recebido em 21/05/08 Versão reformulada recebida em $29 / 07 / 08$ Publicado em 15/04/09 